\title{
Reflets
}

Revue d'intervention sociale et communautaire

\section{Le Cadre de référence sur l'appropriation du pouvoir d'agir personnel et collectif}

\section{Liliane Bertrand}

Volume 17, numéro 1, printemps 2011

URI : https://id.erudit.org/iderudit/1005239ar

DOI : https://doi.org/10.7202/1005239ar

Aller au sommaire du numéro

Éditeur(s)

Reflets, Revue d'intervention sociale et communautaire

ISSN

1203-4576 (imprimé)

1712-8498 (numérique)

Découvrir la revue

Citer cet article

Bertrand, L. (2011). Le Cadre de référence sur l'appropriation du pouvoir d'agir personnel et collectif. Reflets, 17(1), 191-202. https://doi.org/10.7202/1005239ar

Tous droits réservés (C) Reflets, Revue d'intervention sociale et communautaire, 2011
Cocument est protégé par la loi sur le droit d'auteur. L'utilisation des services d'Érudit (y compris la reproduction) est assujettie à sa politique d'utilisation que vous pouvez consulter en ligne.

https://apropos.erudit.org/fr/usagers/politique-dutilisation/ 


\section{Le Cadre de référence sur l'appropriation du pouvoir d'agir personnel et collectif}

Liliane Bertrand

Organisatrice communautaire retraitée, CSSS de Gatineau-CLSC de Hull

En novembre 2007, une équipe de travail ${ }^{1}$ a élaboré le Cadre de référence sur l'appropriation du pouvoir d'agir personnel et collectif, lequel s'adresse aux intervenantes et aux intervenants désireux

- de répondre adéquatement à l'urgence ou à l'intensité d'une situation;

- de diminuer les facteurs de risque en développant des réseaux sociaux de soutien et de solidarité, de saines habitudes de vie et en renforçant le tissu social et communautaire;

- de bâtir des facteurs de robustesse en instaurant des structures sociales adéquates et en prévenant l'apparition ou la résurgence de problèmes sociaux, économiques, physiques et politiques.

Le présent article présente trois des éléments du Cadre de référence sur l'appropriation du pouvoir d'agir personnel et collectif, à savoir, l'Analogie de la chute, le cadre de référence proprement dit et des répliques de saynètes ${ }^{2}$. Un paragraphe portant sur la métaphore de la descente et la montée de l'escalier en lien avec le pouvoir d'agir et un autre sur les conditions d'application du cadre complètent notre propos. 


\section{L'Analogie de la chute}

Avant de lire l'ensemble du Cadre de référence sur l'appropriation du pouvoir d'agir personnel et collectif, il est intéressant de nous interroger sur notre intervention, de la situer par rapport à l'Analogie de la chute et de s'inspirer de cette dernière. Agissonsnous dans la première section de l'Analogie, celle de la réaction aux situations vécues par nos concitoyens? Agissons-nous en collaborant à la construction d'un tissu social solidaire? Agissonsnous en travaillant à l'élaboration de structures sociales visant un changement global?

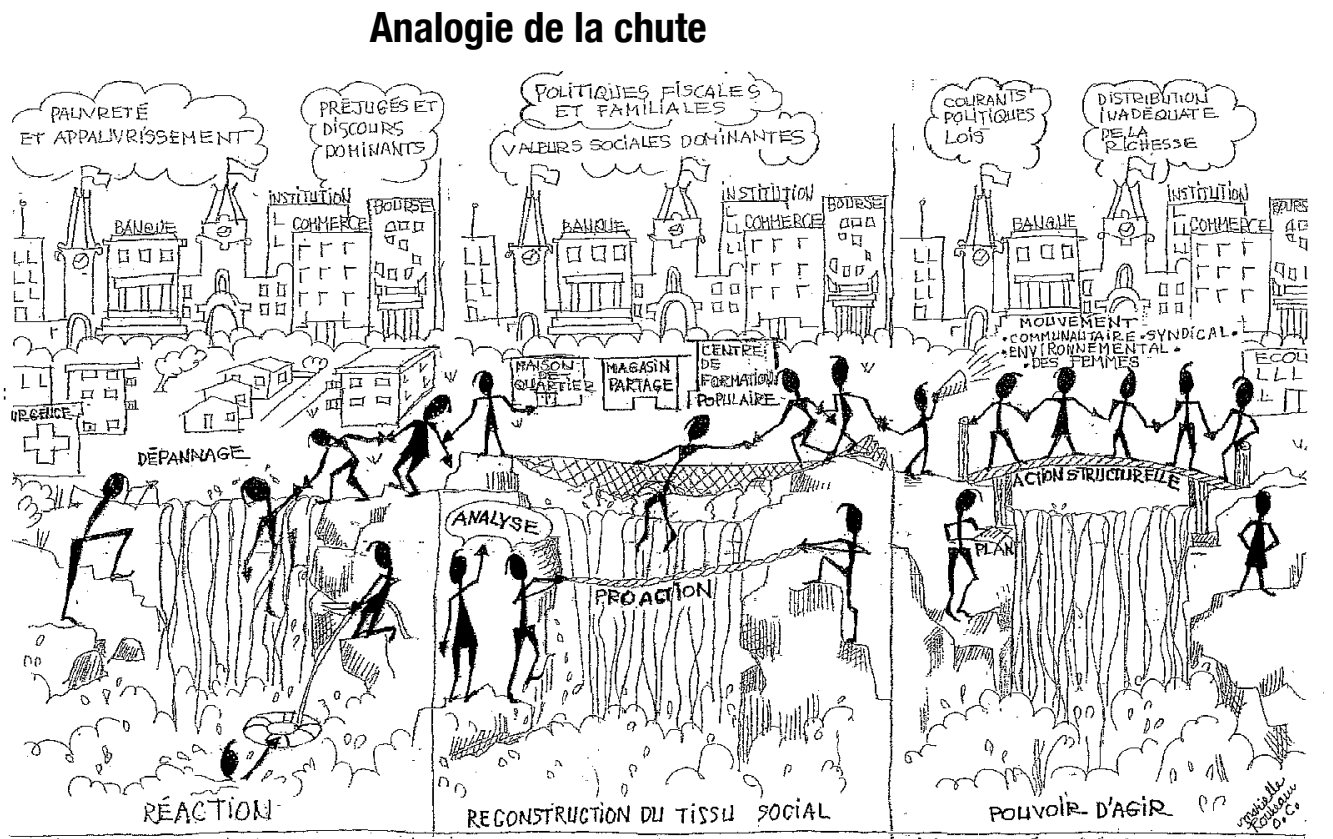

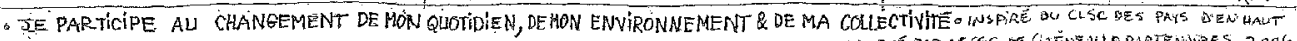

L'Analogie de la chute souligne la nécessité d'un travail à relais entre nos interventions respectives afin d'arriver à un changement social et l'importance de la vision à long terme qui doit accompagner tout processus d'appropriation du pouvoir d'agir. Et à la fin de la lecture du cadre de référence, nous sommes en mesure de voir si notre intervention se situe toujours dans la même section de 
l'Analogie de la chute et de prévoir des façons de collaborer avec les intervenantes ou intervenants des autres sections.

\section{Le cadre de référence}

Notre rôle d'agent de changement social exige de reconnaître les forces et le vécu des personnes, groupes, communautés et collectivités auprès desquelles ou avec lesquelles nous œuvrons et, dans un rapport égalitaire, de prendre en compte leurs droits et leurs besoins. De là, en tant qu'utilisatrices ou utilisateurs du Cadre de référence sur l'appropriation du pouvoir d'agir personnel et collectif, notre emploi des termes " citoyens " ou "citoyennes " plutôt que " clients ", " clientes " ou "bénévoles".

Deux schémas nous aident à intégrer l'essentiel du cadre de référence. Le premier fait clairement état de deux de ses qualités importantes, la diversité et la circularité.

\section{Circularité ... le pouvoir d'agir en mouvement Diversité ... entrées et sorties multiples}

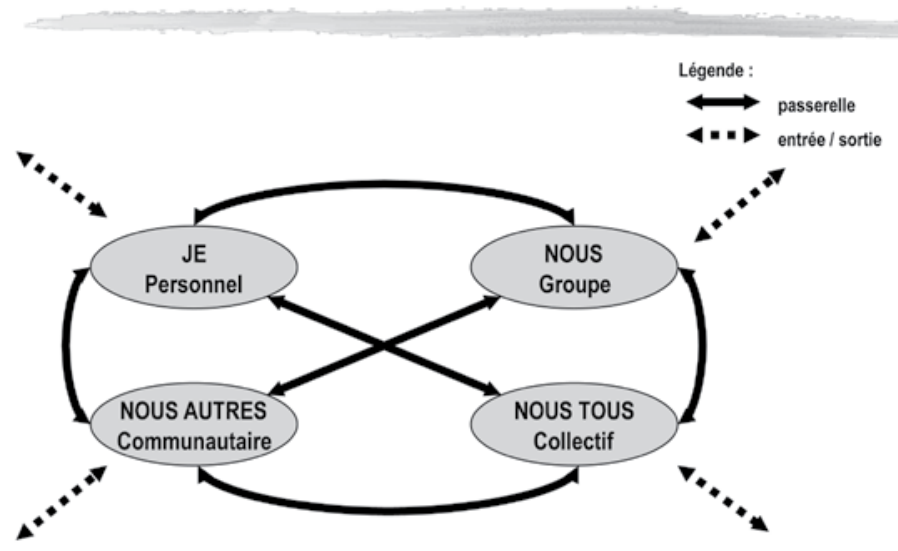

La diversité permet au citoyen d'entrer et de sortir du processus par l'un ou l'autre des quatre volets - personnel, de groupe, communautaire ou collectif. Il n'y a pas d'ordre de préférence, de porte d'entrée principale ou de trajet prédéterminé. 
Par la circularité, le processus d'appropriation du pouvoir d'agir, personnel ou collectif, se veut un processus en mouvement. Les passages entre les différentes perspectives d'intervention peuvent se vivre selon le vécu et les besoins de la personne, du groupe, de la communauté ou de la collectivité. En voici quelques exemples :

- Nous travaillons au développement d'une vie communautaire fière et solidaire dans un quartier appauvri. Dans le cadre d'un projet visant son embellissement, nous rénovons des clôtures avec l'aide des résidents (intervention communautaire). L'une des résidentes profite de notre complicité dans le maniement du marteau pour dévoiler après plus de quarante ans de silence qu'elle a été violée à l'âge de quinze ans. Après l'avoir accueillie dans ce qu'elle partageait de si intime, nous l'avons accompagnée dans son passage vers un travail plus personnel avec une intervenante que nous lui avons présentée. Cette résidente s'appropriait à la fois son pouvoir d'agir communautaire en s'impliquant dans le projet de rénovation des clôtures, et son pouvoir d'agir personnel en allant chercher le soutien nécessaire à la suite de la révélation de son secret (intervention personnelle).

- Nous profitons d'une manifestation (intervention collective) chapeautée par un organisme de défense de droits pour discuter à l'occasion d'un café-rencontre de l'enjeu social sous-jacent (intervention de groupe). Les quelques citoyens nouvellement conscientisés, intéressés et disponibles, nous accompagnent à la manifestation (intervention collective).

- Avec des partenaires représentant des organismes ou autres décideurs, nous travaillons à la mise sur pied d'activités visant le développement de saines habitudes de vie (interventions individuelles, de groupe et communautaires). Nous conscientisons chacun d'eux à l'importance d'utiliser son pouvoir d'influence pour modifier des politiques sociales en cours (intervention collective).

Le second schéma présente les étapes qui sont cœur de l'action et que chaque intervenante ou intervenant a la responsabilité de franchir. Il présente aussi de manière succincte les différentes perspectives d'intervention. 


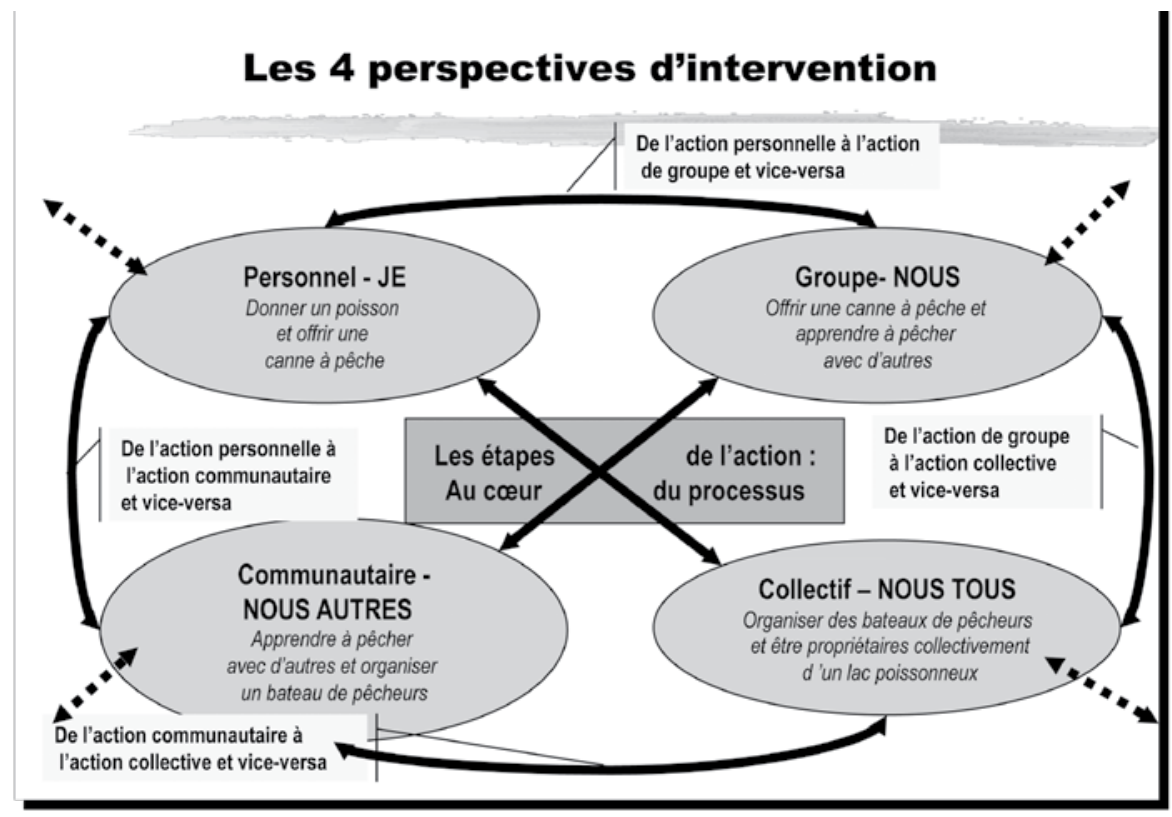

Parce qu'il préconise symboliquement une meilleure répartition de la richesse, le "droit à un lac poissonneux " est une métaphore essentielle au Cadre de référence sur l'appropriation $d u$ pouvoir d'agir personnel et collectif.

\section{Les répliques des saynètes}

Des saynètes mettent en scène Bonhomme allumette, le personnage principal de l'Analogie de la chute. Joué par des citoyennes ou citoyens, ce texte permet d'alimenter la discussion sur l'appropriation du pouvoir d'agir et le changement social. Son seul défaut est de présenter le processus d'une manière linéaire qui, tout en demeurant plausible, n'est pas exclusive. Les équipes intéressées pourraient inventer de nouvelles saynètes qui permettraient de comprendre qu'il est possible d'entrer dans le processus d'appropriation du pouvoir d'agir à partir d'une 
perspective d'intervention autre que personnelle (de groupe, communautaire ou collective). D'autres saynètes pourraient mettre en scène des citoyennes ou des citoyens qui choisissent d'entrer dans le processus et en ressortir sans passer par un autre niveau d'implication.

\section{Biais choisi par l'équipe de conception}

Le Cadre de référence sur l'appropriation du pouvoir d'agir personnel et collectif met l'accent sur la descente et la montée de l'escalier, dont l'illustration aide à comprendre le biais choisi par l'équipe de conception.

La descente de l'escalier traite de la perte de pouvoir d'agir que suscite une distribution inadéquate de la richesse ainsi que les conditions négatives que cela entraîne.

\section{Principes qui soutiennent la vision : La descente de l'escalier}

\section{La distribution inadéquate de la richesse crée des} problématiques d 'inégalités sociales, de pauvreté et d'exclusion

Ces conditions de vie négatives créent des problèmes sociaux (violence, isolement, santé, etc.)

Ceux-ci engendrent une perte de pouvoir d 'agir des personnes et des communautés sur leurs conditions de vie

Inégalités sociales $\Rightarrow$ Conditions de vie négatives $\Rightarrow$ Problèmes sociaux $\Rightarrow$ Perte de pouvoir d 'agir 
La montée de l'escalier montre comment l'appropriation du pouvoir d'agir, personnel ou de groupe, favorise à court terme l'amélioration des conditions de vie des citoyens, transforme les structures sociales et réduit les inégalités. En retour, cette réduction des inégalités et cette transformation des structures sociales engendrent une augmentation du pouvoir d'agir pour l'ensemble de la collectivité.

\section{Principes qui soutiennent la vision : La montée de l'escalier}

L'appropriation du pouvoir d'agir personnel et de groupe peut permettre l'amélioration des conditions de vie immédiates (court terme)

L'amélioration des conditions de vie et l'appropriation du pouvoir d'agir des communautés peuvent transformer le tissu social ou les structures sociales et réduire les inégalités (long terme)

La réduction des inégalités et la transformation des structures sociales engendrent l'augmentation du pouvoir d 'agir pour I 'ensemble de la collectivité

Augmentation du pouvoir d 'agir $\Rightarrow$ Meilleures conditions de vie $\Rightarrow$ Réduction des inégalités et transformation des structures $\Rightarrow$ Augmentation du pouvoir d'agir collectif

\section{Conditions essentielles à l'application du cadre de référence}

\section{Croire que le changement social est possible}

Si nous travaillons avec une personne victime de violence et que nous ne croyons pas que sa démarche personnelle, le développement de son nouveau réseau, son affirmation et la solidarité avec d'autres victimes peuvent faire la différence, 
pourquoi alors intervenir? Cette remise en question s'applique à toutes les réalités vécues par les citoyennes ou citoyens, par les communautés et par les collectivités.

\section{Reconnaître les droits économiques, sociaux et culturels}

Nous vivons dans une société de droit. Or, quand les citoyennes et les citoyens sont appauvris, le courant social nous invite en tant que voisines, voisins, enseignantes, enseignants, intervenantes et intervenants sociaux à agir comme si c'était eux le problème et à les aider à modifier leurs comportements.

Nos institutions veulent trop souvent que nous agissions pour solutionner à court terme le problème d'une personne en particulier. Nous devons diminuer les listes d'attente, mais nous ne travaillons presque plus à éviter que les personnes et les générations à venir aient à vivre le même problème. Il importe de travailler avec les personnes à développer des conditions qui amélioreront leur vie, mais il faut aussi reconnaitre avec elles que d'autres vivent des situations inacceptables dans une société qui a pourtant signé le Pacte international des droits économiques, sociaux, et culturels (PIDESC) en vigueur depuis 1976.

Avec le PIDESC, le Canada et le Québec reconnaissent à chaque citoyen le droit à la santé, à l'éducation, à un logement, à la sécurité alimentaire et à un revenu décent. Pourtant combien d'actions sociales et communautaires contribuent seulement à permettre à des citoyens de vivre un peu mieux, mais toujours dans la pauvreté? Comment se fait-il que lorsque des groupes manifestent pour témoigner du droit au logement ou à un revenu décent les médias et l'opinion publique les considèrent-ils comme des fauteurs de troubles?

Plus nous travaillerons à conscientiser nos concitoyennes et concitoyens sur leurs droits fondamentaux, plus nous sortirons de l'intervention d'aide temporaire pour aller tout doucement vers l'entraide et la solidarité à long terme. Ainsi, nous pourrons travailler collectivement à la réduction des inégalités et à la transformation des structures sociales. 


\section{Reconnaître les forces des citoyens}

Pour réussir à appliquer le Cadre de référence sur l'appropriation du pouvoir d'agir personnel et collectif, il est fondamental de croire à la force qui habite chaque citoyenne ou citoyen. Les Maisons de Quartier du comité Inter-Quartiers de Gatineau ont adopté un cadre de référence dans lequel elles traitent de trois concepts clés : l'accueil, l'accompagnement et l'invitation.

L'accueil : les citoyennes ou citoyens ne sont pas des clients ou des problèmes ambulants. Ces personnes se retrouvent face à nous avec leurs histoires, leurs habiletés, leurs forces et leurs besoins. En les accueillant avec toute leur force et toute leur vulnérabilité, nous serons en tant qu'intervenantes ou intervenants nécessairement respectueux envers elles. Nous verrons en elles des personnes entières et non des problèmes à régler;

L'accompagnement : toujours en tant qu'intervenantes ou intervenants, si nous sommes confrontés à la faim vécue par des personnes ou des familles, il importe que nous puissions les dépanner tout en préservant leur dignité. Nous n'avons pas à les juger, mais nous devons plutôt exprimer notre compréhension et notre conscience du non-respect de leurs droits économiques. Nous devons leur rappeler qu'elles ne sont pas seules et pas coupables de ce qui leur arrive.

Avec le Cadre de référence sur l'appropriation du pouvoir d'agir personnel et collectif, nous leur proposerons de se joindre à d'autres afin de développer des solutions collectives visant à répondre à des besoins collectifs - cuisine collective, jardins communautaires, etc. Qui plus est, nous les informerons sur l'existence de telle ou de telle ressource, allant même jusqu'à les y conduire et à personnaliser le contact en leur présentant une intervenante ou un intervenant;

L'invitation : pour favoriser l'appropriation du pouvoir d'agir, il est très important d'inviter les personnes avec lesquelles nous travaillons à participer à une activité communautaire. Nous 
inviterons les citoyennes ou les citoyens, le groupe, la communauté ou la collectivité à s'impliquer en identifiant les forces que nous leur connaissons, en évoquant la reconnaissance de leurs droits, en les conscientisant à la place du "je ", du "nous ", du "nous autres » et du "nous tous » et en respectant leur histoire actuelle et donc, en respectant leur rythme ainsi que leur désir de poursuivre ou non le processus.

\section{Développer des rapports égalitaires}

Pour appliquer le Cadre de référence sur l'appropriation du pouvoir d'agir personnel et collectif, nous devons nous rappeler en tant qu'intervenantes ou intervenants que la personne qui est devant nous possède une expérience particulière et une richesse qui lui sont propres. Elle nous apprend une réalité et, très souvent, une survivance qui nous sont étrangères. Elle est plus puissante qu'elle ne le croit, parce que trop fréquemment les normes sociales en cours n'ont jamais valorisé ses forces.

\section{Reconnaître l'importance de la parole}

Les intervenantes ou intervenants qui désirent mettre en application le Cadre de référence sur l'appropriation du pouvoir d'agir personnel et collectif acceptent de prendre la parole pour nommer ce en quoi ils croient, même quand cela apparait difficile, et acceptent que la citoyenne ou le citoyen en fasse tout autant. Ils acceptent de vivre l'interinfluence que sous-tend la communication en restant ouverts, mais aussi centrés sur ses valeurs et sur les conditions gagnantes du cadre de référence.

\section{S'indigner devant ce qui est indécent}

Comme intervenantes ou intervenants, il faut profiter de toutes les occasions possibles pour contribuer à un changement positif pour tous. Nos concitoyennes et nos concitoyens prendront exemple de notre argumentaire pour se conscientiser et en conscientiser d'autres. Ensemble, nous pourrons non seulement lutter contre ce qui est indécent, mais aussi nous engager dans un projet social qui vise un monde plus juste avec une répartition équitable de 
la richesse. Notre indignation se transformera en créativité et en stratégies d'influence.

\section{Dresser un bilan de nos actions et célébrer nos succès}

Pour faciliter l'intégration de l'appropriation du pouvoir d'agir et faciliter le passage d'une perspective d'intervention à une autre, il est essentiel de dresser le bilan de nos actions et de célébrer nos succès.

\section{Conclusion}

Collaborer à la réalisation du Cadre de référence sur l'appropriation $d u$ pouvoir d'agir personnel et collectif m'a permis comme intervenante de nommer une pratique que je vivais depuis plusieurs années sans jamais en reconnaitre de fondement théorique. Cet outil, dont certains éléments font l'objet de cet article, m'a été fort utile pour défendre et promouvoir une vision de l'intervention, laquelle menaçait parfois nos organismes.

Comme il est écrit dans le Cadre de référence sur l'appropriation du pouvoir d'agir personnel et collectif, " la citoyenneté est au cœur du processus d'appropriation du pouvoir d'agir... Quelle que soit l'entrée de la personne dans ce processus, l'objectif poursuivi est le changement social en fonction du bien commun, c'est-à-dire un bien pour NOUS TOUS".

Grâce à ce document,j'ai pu faire valoir la pertinence d'actions qui, au lieu de se centrer sur l'individualisation des problèmes, portent sur la responsabilité de nos choix sociaux, économiques et politiques. Les années 2010-2011 m'auront fascinée et interpellée par le fait que notre pays a utilisé des mesures drastiques pour arrêter les manifestations au G20 de Toronto en même temps qu'il appuie l'importance et la richesse du soulèvement populaire de ce que nous appellerons dorénavant le "Printemps arabe".

L'application du Cadre de référence sur l'appropriation du pouvoir d'agir personnel et collectif est une ode à l'importance de la parole 
citoyenne et de la diminution de l'écart entre les riches et les pauvres. Avec ce cadre, chaque citoyenne ou citoyen désireux de s'impliquer dans sa communauté peut se donner des outils pour agir à titre d'actrice sociale ou d'acteur social, à la fois respectueux et solidaire d'un projet social équitable.

Ensemble, il importe que nous puissions continuer à réfléchir à des mécanismes d'intégration de ce cadre de référence au quotidien afin de faciliter les passages d'une perspective d'intervention vers une autre, qu'elle soit du "Nous autres " au "Je » ou du "Nous " $\mathrm{au}$ « Nous tous».

\section{Notes}

1 Jean-Pierre Deslauriers de l'Université du Québec en Outaouais, Mario Dion du Diocèse de Gatineau, Dominic Bergeron, Liliane Bertrand, Lise Hébert et Anne-Renée Simoneau du Centre de Santé et de Services sociaux de Gatineau et Joscelyne Levesque du Regroupement des OSBL d'habitation et d'hébergement avec support communautaire de l'Outaouais.

2 Pour plus de renseignements sur l'ensemble du document, on peut consulter Pratiques sociales, No 15 , une publication du Centre de recherche en intervention sociale (CÉRIS). 